Produto \& Produção, vol. 15 n.1, p. 01-09, fev. 2014

RECEBIDO EM 05/06/2012. ACEITO EM 21/05/2013.

\title{
Mudança de regime Markoviano aplicado à fase I do CEP em ambientes de múltiplos setups
}

\author{
Andre Luis Korzenowski \\ Professor Assistente do Departamento de Estatística FAMAT/PUCRS \\ andre.korzenowski@gmail.com \\ Carla Schwengber ten Caten \\ Programa de Pós-Graduação em Engenharia de Produção e Transportes - UFRGS \\ tencaten@producao.ufrgs.br
}

\section{RESUMO}

O controle estatístico do processo (CEP), através de suas ferramentas, tem como objetivo apontar quando o processo passa de um estado sob controle para um estado fora de controle. O CEP é dividido em duas fases: a fase de diagnóstico sobre a estrutura dos dados (Fase I) e a fase de controle do processo (Fase II). Um dos objetivos principais da Fase I é estimar os valores dos parâmetros do processo. A estimação destes parâmetros fica prejudicada em ambientes de produção flexível, pois as séries são relativamente mais curtas, impedindo uma adequada inferência dos parâmetros. $O$ objetivo deste artigo é apresentar a aplicação de um modelo de mudança de regime markoviano para diagnóstico na Fase I do CEP em ambientes de múltiplos setups. O procedimento de mudança de regime markoviano é capaz de estimar os parâmetros das séries em cada regime, levando em consideração a estrutura de variabilidade comum a todos os regimes a partir da análise da característica da qualidade mensurada.

Palavras-Chave: Fase I, Mudança de Regime Markoviano, múltiplos setups.

\section{ABSTRACT}

The statistical process control (SPC), through its tools, aims to point out when the process is under control of a state to a state out of control. The SPC is divided into two phases: diagnostic data (Phase I) and control phase (Phase II). The main objective of Phase I is to estimate the process parameters. The estimation of these parameters is impaired in a flexible production environment. The series are shorter preventing an accurate inference of process parameters. The aim of this paper is to present the use of Markov switching change model for diagnosis in Phase I of the SPC in environments of multiple setups. The procedure for Markov switching change is able to estimate the parameters of the series in each system, taking into account the variability structure common to all systems from the analysis of the quality characteristic output.

Keywords: Phase I, Markov Switching, multiple setup.

\section{Introdução}

O controle estatístico do processo (CEP) aplicado na indústria, para monitoração de processos produtivos, consagrou-se na década de 30, quando Shewhart (1934) publicou seu trabalho sobre cartas de controle. Desde então, estas cartas vem auxiliando no aperfeiçoamento de produtos e controle de 
aspectos de qualidade. O sucesso na aplicação destes métodos básicos nos dados do processo, segundo Staudhammer et al. (2007), está no atendimento a três suposições: independência, homogeneidade de variâncias e normalidade, sendo esta última especialmente crítica no caso de carta para valores individuais.

O papel dos gráficos de controle, segundo Montgomery (2004), é apontar quando o processo passa de um estado sob controle para um estado fora de controle. Em geral, um processo entra em estado fora de controle quando é acometido por alguma causa especial, seja na variação ou no nível da série. Em geral, os gráficos para cada um destes parâmetros são construídos separadamente, apesar de já haver referências na literatura de gráficos que monitoram conjuntamente os dois parâmetros (ZHOU et al., 2010; ZHANG et al., 2009; HAWKINS \& DENG, 2009; WU et al., 2009; COSTA \& HAHIM, 2006; HE \& GRIGORYAN, 2006). Outra condição básica para implantação de sistemas de controle de qualidade é a de que o processo seja estável, isto é, que a série de dados observada seja estacionária.

A implantação de cartas de controle se dá em duas fases distintas: a fase de diagnóstico sobre a estrutura dos dados, denominada Fase I, e a fase de controle do processo propriamente dita, denominada de Fase II (ZHANG et al., 2009). Como os métodos de controle foram desenvolvidos há muito tempo, um conceito prioritário era mantê-los simples. Entretanto, segundo Windfeldt \& Bisgaard (2009), no ambiente atual de softwares e computadores, o conceito de simples sofreu mudanças fundamentais e com os atuais recursos é possível inserir métodos mais avançados nas Fases I e II. Estes métodos podem ajudar os engenheiros da qualidade com informações preciosas sobre os processos além de permitir muito mais investigações do que apenas as que são efetuadas para verificar as suposições quando usa-se cartas de controle tradicionais.

O objetivo principal da Fase I, segundo Zhang et al. (2009) é avaliar a estabilidade do processo, tratar causas especiais de variação detectadas e estimar os valores dos parâmetros do processo. Em contraste, segundo o mesmo autor, a Fase II tem por principal objetivo a rápida detecção de mudanças no nível dos parâmetros do processo controlado, que foram estimados na Fase I. A avaliação do desempenho das cartas de controle também utiliza duas métricas distintas, associadas, de certo modo, a cada fase de implantação: $\mathrm{o} \mathrm{ARL}_{0}$, que mede o número médio de observações até a observação de um alarme falso; e o $\mathrm{ARL}_{1}$, que mede o numero médio de observações até a detecção de uma observação fora de controle, após o processo passar a esta condição.

Uma vez que os parâmetros do processo são conhecidos a priori, não é necessário a execução de uma Fase I e a Fase II é iniciada imediatamente. Entretanto, em aplicações reais, tem-se que estimar os parâmetros do processo em séries que podem conter alterações de nível, tendências ou ainda outliers devido a presença de variações no processo causadas por causas especiais de variação. Boyles (2000) apresenta resultados da análise da Fase I em um processo autocorrelacionado e aponta que os estimadores dos parâmetros do processo são tendenciosos. Alterações no $\mathrm{ARL}_{0}$ nas cartas $\bar{X}$ e $\mathrm{S}$ foram demonstrados por Korzenowski \& Werner (2012) quando da violação à suposição de normalidade.

Segundo Zhu et al. (2007), as cartas de controle da qualidade são bem sucedidas quando aplicadas em processos industriais de produção em massa, entretanto, atualmente produtos de ciclo curto ou produzidos em pequenos lotes tem criado grandes mudanças na aplicação do CEP. Esta alteração nos tamanhos de lote é motivada pela implantação de sistemas estratégicos de produção focados em produtos e serviços personalizados. Esta estratégia é denominada de Customização em Massa (PINE et al. 1993). Segundo o autor, Customização em Massa tem se mostrado como uma vantagem competitiva importante em vários setores chaves da economia mundial, como automotivo, vestuário e de componentes eletrônicos.

Em ambientes de produção de itens customizados, ou que apresentam flexibilidade na produção de itens, ainda existem preocupações em relação a como implementar as ferramentas de controle da qualidade. Isto por que, em geral, as séries são curtas e os itens em produção estão em constantes mudanças (HO \& TRINDADE, 2009). De fato, de acordo com Fogliatto et al. (2012), esta é uma questão que mantém-se aberta: Como adaptar os esquemas tradicionais de controle de qualidade de modo a considerar características com especificações variáveis? Fogliatto et al. (2012) afirmam não ter conhecimento de publicações na literatura que apresentem uma solução consolidada. Entretanto, indicam que o primeiro passo para construir estas ferramentas é identificar as características da qualidade mensuráveis em todos os produtos e/ou processos. A segunda etapa é desenvolver métodos 
de controle que sejam capazes de considerar conjuntamente estas características com especificações variáveis.

Para atender a demanda pelo monitoramento de pequenos lotes, novos gráficos, tais como de start-up ou de short runs tem sido propostos na literatura (PETRICK et al., 2010; HO \& TRINDADE, 2009; TSIAMYRTZIS \& HAWKINS, 2008; NENES \& TAGARAS, 2007; ZHU et al., 2007; LIAN et al., 2006; ZANTEK, 2006; TSAI et al., 2005). A violação de suposições e dificuldades para estimar os parâmetros do processo continua sendo um fato nestas proposições. Neste contexto as séries são curtas demais para permitir uma boa verificação ou mesmo um bom ajustamento de modelos durante a Fase I, o que implica na implantação do CEP sem o volume de informações necessárias. De acordo com Zantek (2006), esta prática acaba por elevar a taxa de alarmes falsos (associado ao $\mathrm{ARL}_{0}$ ) bem como o $\mathrm{ARL}_{1}$. Jensen et al. (2006) apresentam uma revisão de literatura sobre os efeitos da estimação dos parâmetros nas medidas de desempenho de cartas de controle. Os resultados apresentados pelos autores corroboram as afirmações de Zantek (2006). Além disso, a implantação destas ferramentas não atendem ao presuposto do monitoramento conjunto de características da qualidade oriundas de diversos produtos, tal qual sugerido por Fogliatto et al. (2012), fazendo com que haja a necessidade de se implantar tantas cartas quantos produtos em produção.

Em processos de produção flexíveis de pequenos lotes, a série dos múltiplos produtos avaliada sequencialmente sofre alterações de forma determinística, pois envolvem a alteração intencional do processo pelas mudanças de configuração dos equipamentos na linha de produção. A série não será estacionária, pois, no mínimo, existem mudanças de nível na série em função da característica de qualidade nominal de cada produto. Neste caso, pode-se dizer que o processo apresenta diferentes estados, associados aos diferentes produtos em produção. Assim, uma série de dados oriundos de um processo deste tipo poderia ser representado por

$x_{1, k_{\{j\}}}, x_{2, k_{\{j\}}}, \ldots, x_{t, k_{\{j\}}}, \ldots, x_{t+1, k_{\{l \neq j\}}}, \ldots, x_{t+i, k_{\{l \neq j\}}}, x_{t+i+1, k_{\{m \neq l\}}}, \ldots t=1,2, \ldots ; k=1, \ldots, p$

onde $x_{t, k}$ é o vetor das observações da característica da qualidade, $k$ indica qual produto está em produção no tempo $t$ e $j$ o tamanho de lote desconhecido, onde $j \in\{1,2, \ldots\}$.

Este artigo foca na análise retrospectiva (ou Fase I) da implantação de cartas de controle e, portanto, não serão apresentados resultados referentes ao monitoramento do processo (Fase II). O objetivo é propor a utilização do modelo de mudança de regime markoviano para diagnóstico na Fase I do controle de uma característica de qualidade em ambientes flexíveis ou customizados. O procedimento de mudança de regime markoviano ajusta um modelo matemático para cada estado do processo (para cada tipo de produto). Além disso, determina a probabilidade de que, em um dado instante do tempo, o processo esteja operando em cada um dos regimes. A acertiva do sistema de mudança de regime markoviano apresentará a qualidade do ajuste, uma vez que as mudanças observadas nas probabilidades dos estados devem ser coincidentes com as alterações efetuadas nas configurações dos equipamentos.

A metodologia sugerida é capaz de estimar os parâmetros da série em cada regime, levando ainda em consideração a estrutura de variabilidade comum a todos os regimes (variabilidade devida à máquina, operador, lote de matéria prima, etc.). Além disso, supõe-se que pequenas corridas em cada regime podem ser estimadas, o que traria aplicação em ambientes de customização em massa ou lean factoring, onde, de acordo com Fogliatto et al. (2012), as séries produtivas de cada tipo de produto podem ser relativamente curtas ou até mesmo unitárias. Estes parâmetros estimados poderiam ser utilizados na construção das cartas na Fase II, onde uma nova geração de cartas de monitoramento do processo necessitam ser desenvolvidas e aplicadas. Uma sugestão é a utilização de cartas de resíduos ao modelos ajustados através do sistema de mudança de regime markoviano.

A contribuição deste artigo está na aplicação desta metodologia em um processo de produção de múltiplos itens, destacado na literatura recente como um ambiente carente de soluções e fruto de preocupações no meio acadêmico. Uma série de dados reais de produção em uma empresa produtora de bobinas de filme plástico foi utilizada para demonstração do procedimento. Assim, a segunda seção apresenta os pressupostos teóricos do modelo de mudança de regime markoviano. A seção 3 apresenta os procedimentos metodológicos adotados para a implantação no banco de dados de exemplo e esta seção é seguida dos resultados encontrados. A última seção traz considerações finais e sugestões de pesquisas futuras. 
Segundo Kim \& Nelson (1999), um processo de Markov é um processo estocástico clássico no qual a variável aleatória $X_{t}$ possui uma relação de dependência particular no tempo. A característica especial do modelo markoviano é a hipótese de que a realização do regime $S_{t}=1, \ldots, k$ não observado é determinado por um processo estocástico markoviano no estado discreto e tempo discreto, que é definido pelas probabilidades de transição.

A probabilidade de $X_{t+1}$ estar no estado $j$ no momento $t+1$ dado que $X_{t}$ está no estado $i$ no momento $t$, denominada de probabilidade de transição de um passo, é definida por:

$P_{i j}^{t, t+1}=\operatorname{Pr}\left\{X_{t+1}=j \mid X_{t}=i\right\}$

Como pode ser visto, a probabilidade de transição $P_{i j}^{t, t+1}$ é não apenas função do estado, mas também do tempo de transição. Por outro lado, se $P_{i j}^{t, t+1}$ for independente do tempo, então o processo de Markov tem uma probabilidade de transição estacionária, e $P_{i j}^{t, t+1}=p_{i j}$. Como podem existir $k$ estados, as probabilidades de transição entre estes estados podem ser representadas através de uma matriz de probabilidade de transição $P=\left[p_{i j}\right] \in M_{\{k x k\}}$ tal como:

$P=\left[\begin{array}{ccc}p_{11} & \cdots & p_{1 k} \\ \vdots & \ddots & \vdots \\ p_{k 1} & \cdots & p_{k k}\end{array}\right]$

onde: $\sum_{j=1}^{k} p_{i j}=1$ para $i=1,2, \ldots, k, p_{i j} \geq 0$ para $i, j=1,2, \ldots, k$.

Seja o vetor de observações $y_{t}=\left(y_{i t}, \ldots, y_{n t}\right)^{\prime}, y \in R^{n}, \square=1, \ldots, T$ e os diferentes estados não observados em que a variável pode se encontrar dado por $S_{t} \in 1, \ldots, k$. Supondo uma função de distribuição para a variável $y_{t}$ :

$f\left(y_{t} \mid S_{t=j} ; \Theta\right)=\frac{1}{\sqrt{2 \pi \sigma^{2}}} \exp \left\{\frac{-\left(y_{t}-\mu_{j}\right)^{2}}{2 \sigma_{j}^{2}}\right\}, j=1,2, \ldots, k$

então tem-se que $y_{1 t} \sim N\left(\mu_{1}, \sigma_{1}\right)$, se o processo está no regime um, $y_{2 t} \sim N\left(\mu_{2}, \sigma_{2}\right)$ se o processo está no regime dois, e assim sucessivamente até o regime $k$, onde o vetor de parâmetros do modelo é dado por $\Theta=\left(\mu_{1}, \sigma_{1}, \mu_{2}, \sigma_{2}, \ldots \mu_{k}, \sigma_{k},\right)$. Note que, se o regime em que o processo encontra-se no tempo $t$ para todo $t \in\{1,2 \ldots, T\}$ é conhecido a priori, então pode-se ajustar um modelo com variáveis dummy. Entretanto, quando $S_{t} \in\{1, \ldots, k\}$ não é observável, necessita-se determinar o logaritmo da função de verossimilhança considerando a densidade conjunta de $y_{t}$ e a variável $S_{t}$ não observável como produto das densidades condicionais e marginais:

$f\left(y_{t}, S_{t} \mid \psi_{t-1}\right)=f\left(y_{t} \mid S_{t}, \psi_{t-1}\right) \times f\left(S_{t} \mid \psi_{t-1}\right)$

onde $\psi_{t-1}$ é a informação até o tempo $t-1$. Após, para obter a densidade marginal de $y_{t}$, integra-se fora a variável $S_{t}$ da densidade conjunta através do somatório de todos os possíveis valores de $S_{t}$ :

$$
\begin{aligned}
f\left(S_{t} \mid \psi_{t-1}\right) & =\sum_{S_{t}=1}^{k} f\left(y_{t}, S_{t} \mid \psi_{t-1}\right) \\
& =\sum_{S_{t}=1}^{k} f\left(y_{t} \mid S_{t}, \psi_{t-1}\right) \times f\left(S_{t} \mid \psi_{t-1}\right) \\
& =\sum_{S_{t}=1}^{k} \frac{1}{\sqrt{2 \pi \sigma_{S_{t}}^{2}}} \exp \left\{\frac{-\left(y_{t}-\mu_{S_{t}}\right)^{2}}{2 \sigma_{S_{t}}^{2}}\right\} \times P\left[S_{t}=j \mid \psi_{t-1}\right]
\end{aligned}
$$

O logaritmo da função de máxima verossimilhança será então dado por:

$\ln L=\sum_{t=1}^{T} \ln \left\{\sum_{S_{t}=1}^{k} f\left(y_{t} \mid S_{t}, \psi_{t-1}\right\}\right) \times P\left[S_{t}=j \mid \psi_{t-1}\right]$

Supondo que a variável $S_{t}$ comporta-se como em um processo markoviano, isto é, independente dos valores de seu próprio passado, então as probabilidades $\operatorname{Pr}\left[S_{t-1}=i \mid \psi_{t-1}\right], i=$ $0, \ldots, k$ podem ser obtidas através do seguinte filtro: 
Passo 1: Dado $\operatorname{Pr}\left[S_{t-1}=i \mid \psi_{t-1}\right] i=0, \ldots, k$, no início da i-ésima iteração, os termos $\operatorname{Pr}\left[S_{t}=\right.$ $\left.j \mid \psi_{t-1}\right], j=0, \ldots, k$ são calculados como

$$
\begin{aligned}
\operatorname{Pr}\left[S_{t}=j \mid \psi_{t-1}\right] & =\sum_{i=1}^{k} \operatorname{Pr}\left[S_{t}=j, S_{t-1}=i \mid \psi_{t-1}\right] \\
& =\sum_{i=1}^{k} \operatorname{Pr}\left[S_{t}=j \mid S_{t-1}=i, \psi_{t-1}\right] \times \operatorname{Pr}\left[S_{t-1}=i \mid \psi_{t-1}\right]
\end{aligned}
$$

onde $\operatorname{Pr}\left[S_{t}=j, S_{t-1}=i\right], i=1, \ldots, k ; j=1, \ldots, k$, são as probabilidades de transição.

Passo 2: Uma vez que $y_{t}$ é observado no final da i-ésima interação, as probabilidades podem ser atualizadas da seguinte forma:

$$
\begin{aligned}
\operatorname{Pr}\left[S_{t}=j \mid \psi_{t}\right] & =\sum_{i=1}^{k} \operatorname{Pr}\left[S_{t}=j \mid \psi_{t-1}, y_{t}\right] \\
& =\frac{f\left(S_{t}=j, y_{t} \mid \psi_{t-1}\right)}{f\left(y_{t} \mid \psi_{t-1}\right)} \\
& =\frac{f\left(y_{t} \mid S_{t}=j, \psi_{t-1}\right) \times \operatorname{Pr}\left[S_{t}=j \mid \psi_{t-1}\right]}{\sum_{j=1}^{k} f\left(y_{t} \mid S_{t}=j, \psi_{t-1}\right) \times \operatorname{Pr}\left[S_{t}=j \mid \psi_{t-1}\right]}
\end{aligned}
$$

Estes passos devem ser iterados a fim de obter $\operatorname{Pr}\left[S_{t}=j \mid \psi_{t}\right]$ para todos $t=1, \ldots, T$.

\section{Aplicação do modelo}

Os dados analisados foram obtidos junto a uma empresa do setor plástico localizada ao sul do Brasil. Esta empresa produz uma série de produtos sob demanda, tendo por base filmes plásticos. Esta demanda é irregular e, portanto, não há tamanhos de lote mínimos produzidos e ou padronizados. Os produtos acabados são oriundos de duas estruturas distintas. Estes itens são produzidos no mesmo processo através de alterações nas configurações do equipamento e itens de matéria prima, sendo mantidas constantes outras fontes de variação. Foram coletadas 306 amostras sequenciais da produção, conforme demanda, em intervalos fixos e observada a característica de qualidade gramatura do filme plástico produzido. Esta é uma característica importante do produto, pois, segundo informações obtidas junto aos técnicos da empresa, a resistência à tração está fortemente relacionada a esta característica.

O modelo ajustado considerou, além de uma constante regressora e um componente autoregressivo, a variabilidade da componente de ruído nos efeitos de mudança de regime markoviano. Assim, o modelo ajustado foi:

$y_{t}=\beta_{S_{t}}+\phi_{1, S_{t}} y_{t-1}+\varepsilon_{t}$

com

$P=\left[\begin{array}{ll}p_{11} & p_{12} \\ p_{21} & p_{22}\end{array}\right]$

onde $S_{t}$ representa o estado no tempo $t$, isto é 1 ou $2 ; \beta_{S_{t}}$ é o coeficiente beta constante, regressor no estado $S_{t} ; \phi_{1, S_{t}}$ é o coeficiente da porção autoregressiva defasada em $t-1$ no estado $S_{t} ; y_{t-1}$ é a observação defasada em $t-1 ; \varepsilon_{t}$ é um vetor de resíduos que supõe-se seja normalmente distribuído com média zero e variância $\sigma^{2} ; P$ é a matriz de probabilidades de transição entre os estados e $p_{i j}$ é a probabilidade de transição do estado $i$ para o estado $j$ em uma etapa, onde $i, j \in\{1,2\}$. Destaca-se que o modelo ajustado na Equação 10 foi escolhido para este particular caso, sendo possível a escolha de um modelo de qualquer forma, de acordo com as características do processo, o que garante a generalidade do método para implantação em diversos sistemas de produção.

A estimativa dos parâmetros nos modelos de mudança de regime markoviano foi efetuada com auxílio do software Matlab 7.5.0 (R2007b) e do pacote desenvolvido por Perlin (2009). Os gráficos foram gerados com auxílio do software R-Project v. 2.11.1. 
A Figura 1 apresenta a série de dados coletada da variável Gramatura. Observa-se a evidência de mudanças de nível na série, como resultado da alteração no setup de produção, além de variabilidades decorrentes do processo de produção.

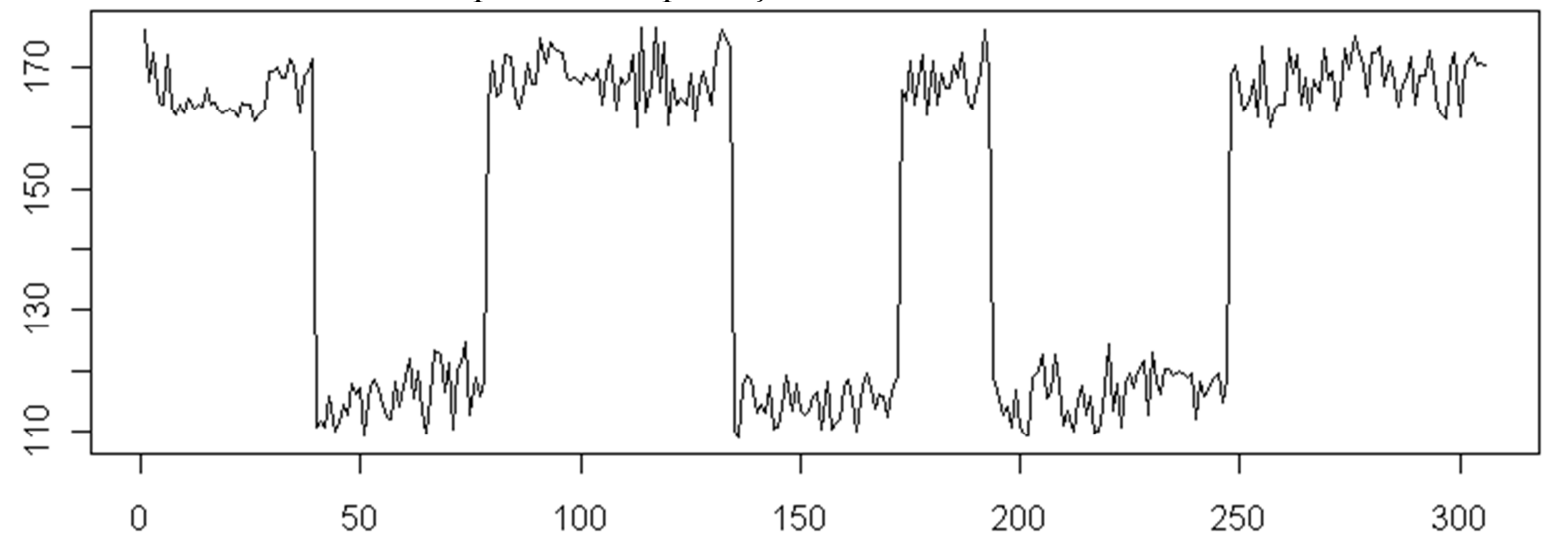
demanda

Figura 1 - Série de gramatura de bobinas de filme plástico em uma linha de produção sob

De acordo com os resultados obtidos, o modelo ajustado obteve convergência. No total, dez parâmetros foram estimados sendo apresentados na Tabela 1. O modelo conseguiu identificar a mudança de nível na série, apontando alterações significativas na constante de cada regime. Do mesmo modo, a variância da componente de ruído é identificada como sendo diferente entre os regimes, o que indica que o processo não apenas apresenta alteração de nível, em função dos ajustes da máquina para a produção dos diferentes produtos, como também as variações inerentes a cada produto neste processo são distintas.

Tabela 1 - Parâmetros finais do modelo de mudança de regime markoviano

\begin{tabular}{|c|c|c|c|c|c|c|}
\hline \multirow{3}{*}{ Parâmetros } & \multicolumn{6}{|c|}{ Estados } \\
\hline & \multicolumn{3}{|c|}{$S_{t}=1$} & \multicolumn{3}{|c|}{$S_{t}=2$} \\
\hline & Coef. & E.P. & p-valor & Coef. & E.P. & p-valor \\
\hline$\sigma_{S_{t}}^{2}$ & 673,34 & 108,98 & $<0,01$ & 1312,65 & Inf & 1,00 \\
\hline Constante & 145,51 & 1,67 & $<0,01$ & 109,17 & 3,73 & $<0,01$ \\
\hline$\phi_{1}$ & 0,0147 & 0,0077 & 0,06 & 0,0137 & 0,0139 & 0,32 \\
\hline
\end{tabular}

A matriz com as probabilidades de transição estimadas foi:

$P=\left[\begin{array}{ll}0,90(0,08) & 0,10(0,07) \\ 0,10(0,08) & 0,90(0,11)\end{array}\right]$

onde tem-se $p_{i j}$ (E.P.). Os resultados mostram probabilidade igual a 0,90 de manter-se em um determinado estado contra probabilidade de 0,10 de trocar de estado. De fato, esta probabilidade não possuí uma aplicabilidade prática no caso de ambientes flexíveis pois as mudanças na linha de produção são conhecidas, isto é, sabe-se exatamente onde foi efetuada a mudança de regime pela alteração na configuração dos equipamentos. Entretanto, é possível obter através das probabilidades de transição o tempo esperado em cada regime. A duração esperada em cada regime foi de 10,32 períodos de tempo em média. Isto significa que o número médio de observações coletadas em cada produto é de 10,32 observações. Esta estimativa pode ser útil para a otimização das cartas para a Fase II, dependendo do tipo de procedimento a ser adotado.

A Figura 2 apresenta as probabilidades filtradas e alisadas do sistema encontrar-se em cada estado em cada período do tempo para o modelo ajustado. Neste caso, observa-se que as probabilidades filtradas de que o sistema encontre-se no estado 2, quando ele realmente encontra-se no estado 2, são relativamente menores do que as probabilidades do sistema estar no estado 1, quando ele realmente está no estado 1. A diferença entre as variabilidades de cada regime gera esta incerteza apresentada pelo modelo. Entretanto, este nível de incerteza sobre em qual estado encontra-se o sistema é de certo modo minimizado quando as probabilidades são alisadas. 


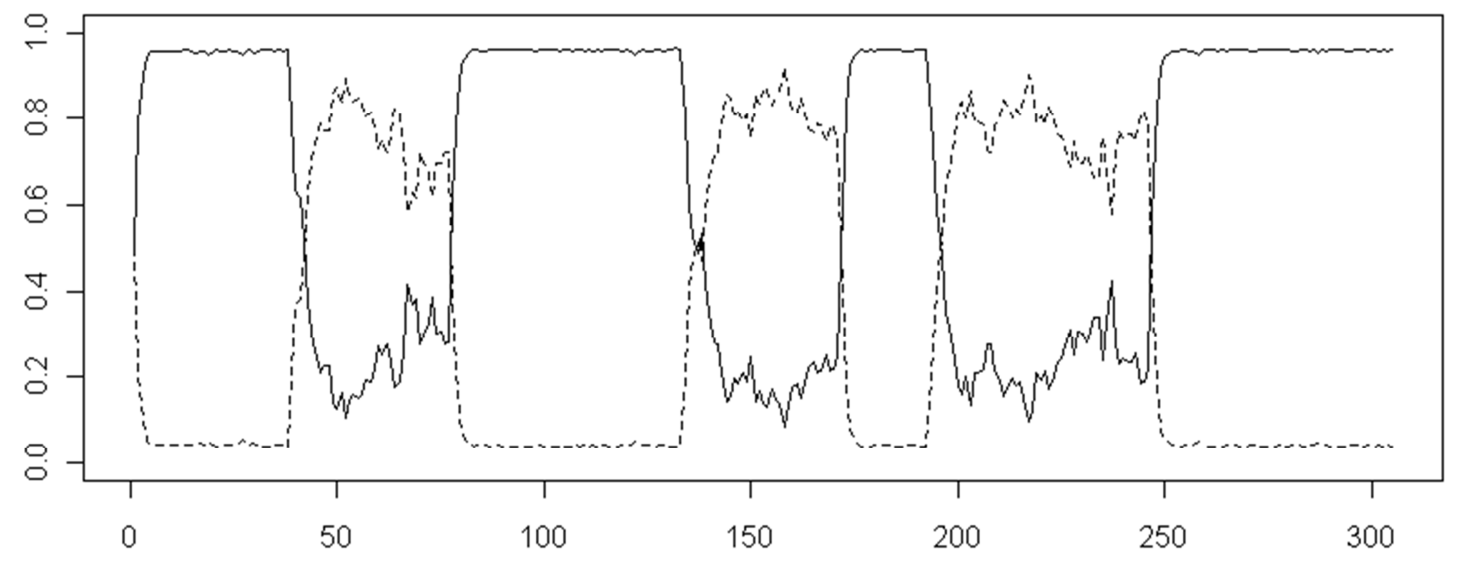

(a)

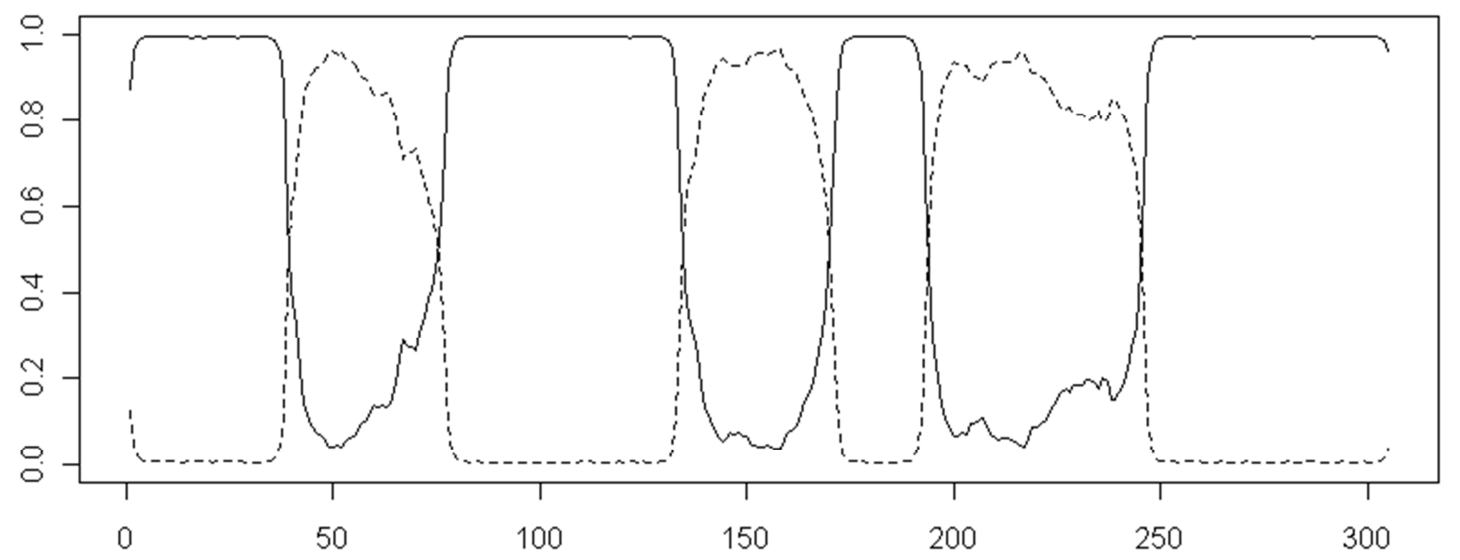

(b)

Figura 2 - Probabilidades filtradas (a) e suavizadas (b) para cada estado

\section{Considerações finais}

Este artigo foca-se na análise retrospectiva da implantação de cartas de controle. Durante a Fase I de implantação do controle da qualidade, importantes aspectos relacionados ao processo precisam ser compreendidos e identificados. Estudos referidos apresentam efeitos gerados pela estimação dos parâmetros de um processo, bem como em relação à violação das suposições das ferramentas de controle. Isto justifica a necessidade de se estabelecer rotinas de implantação do Controle de Qualidade que incluam um bom levantamento de informações na Fase I, associado à análises minuciosas nos dados. Neste aspecto, a utilização de um modelo capaz de diagnosticar com acurácia os parâmetros do processo, e, além disso, que possibilite esse diagnóstico para ambientes flexíveis, ou ainda, para itens customizados, preenche uma lacuna importante existente na literatura acerca da implantação de ferramentas de controle onde os parâmetros do processo não são conhecidos a priori.

A abordagem exploratória, proveniente do ajustamento de modelos de troca de regime markoviano, resulta na capacidade de se avaliar vários aspectos relacionados ao comportamento de um processo múltiplo em um único procedimento. A flexibilidade que este procedimento apresenta em termos da modelagem de diversos parâmetros economiza inúmeras rotinas de implantação. Adotar esta prática para o levantamento de informações em processos multi-etápicos ou no controle da qualidade em ambientes de produção por demanda (puxada) ou ainda de customização em massa, onde o tamanho dos lotes é geralmente pequeno para ajustes tradicionais é a grande contribuição deste trabalho. No caso do controle da qualidade desta empresa de bobinas de filme plástico, a série de Gramatura apresentava certa regularidade em termos de tamanhos de lote. Entretanto as estimativas de tempo em cada regime podem auxiliar em processos de otimização de cartas de controle. 
A verificação da capacidade do modelo em reproduzir os bons resultados aqui encontrados em ambientes com maior número de produtos controlados (maior número de regimes) ou que tenham tamanhos de lotes (sequências) menores e/ou mais variáveis é sugestão para trabalhos futuros. Do mesmo modo, a avaliação da implantação na Fase II de uma carta de resíduos dos modelos ajustados com os parâmetros estimados pelo sistema de mudança de regime markoviano é sugestão para pesquisas futuras.

\section{Referências}

BOYLES, R. Phase I analysis for autocorrelated processes. Journal of Quality Technology, Vol 32, No. 4, p. 395-409, 2000.

COSTA, A. F. B.; RAHIM, M. A. A synthetic control chart for monitoring the process mean and variance. Journal of Quality in Maintenance Engineering, vol. 12, No. 1, p. 81-88, 2006.

FOGLIATTO, F. S.; da SILVEIRA, G. J. C.; BORENSTEIN, D. The mass customization decade: An updated review of the literature. International Journal of production Economics, Vol. 138, 1, p. 14-25, 2012.

HAWKINS, D. M.; DENG, Q. Combined Charts for Mean and Variance Information. Journal of Quality Technology, Vol. 41, No. 4, 2009.

HE, D.; GRIGORYAN, A. Joint statistical design of double sampling X-bar and s charts. European Journal of Operational Research, Vol. 168, p. 122-142, 2006.

HO, L. L.; TRINDADE, A. L. G. Economic design of an X chart for short-run production. Int. J. Production Economics, Vol. 120, p. 613-624, 2009.

KIM, C-J.; NELSON, C. R. State-space models with regime switching: classical and Gibbs-sampling approaches with applications. London: The MIT Press, 1999.

KORZENOWSKI, A. L.; WERNER, L. Probabilidade do erro do tipo I nas cartas X e S de Shewhart sob não normalidade. Produção, Vol. 22, p. 807-816, 2012.

LIAN, Z.; COLOSIMO, B. M.; del CASTILLO, E. Setup Error Adjustment: Sensitivity Analysis and a New MCMC Control Rule. Quality and reliability Engineering International, Vol. 22, p. 403-418, 2006.

MONTGOMERY, D.C. Introduction to statistical quality control. New York: John Wiley \& Sons, 2004.

NENES, G.; TAGARAS, G. The economically designed two-sided Bayesian X- bar control chart. IEuropean Journal of Operational Research, Vol. 183, p. 263-277, 2007.

PERLIN, M. MS_Regress - A Package for Markov Regime Switching Models in Matlab. MATLAB Central File Exchange, $2009 . \quad$ Avaiable in http://www.mathworks.com/matlabcentral/fileexchange/authors/21596

PETRICK, A.; GONSCH, J.; STEINHARDT, C.; KLEIN, R. Dynamic control mechanisms for revenue management with fliexible products. Computers \& Operations research, vol. 37, p. 20272039, 2010.

PINE II, B.J., VICTOR, B., BOYNTON, A.C. Making mass customization work. Harvard Business Review, Vol. 71, No. 5, p. 108-118, 1993.

SHEWHART, W. A. Economic control of quality of manufactured product. New York: D. Van Nostrand Company, 1931.

STAUDHAMMER, C.; MANESS, T. C.; KOZAK, R. A. Profile Charts for Monitoring Lumber Manufacturing Using Laser Range Sensor Data. Journal of Quality Technology, Vol 39, No. 3, 2007. 
TSAI, T-R.; LIN, J-J.; WU, S-J.; LIN, H-C. On estimating control limits of x-bar chart when the number of subgroups is small. Int. J. Advanced Ma- nufacturing Technology, Vol.26, p. 1312-1316, 2005.

TSIAMYRTZIS, P.; HAWKINS, D. M. A Bayesian EWMA Method to Detect Jumps at the Start-up Phase of a Process. Quality and reliability Enginee- ring International, Vol. 54, p. 721-735, 2008.

WINDFELDT, G. B.; BISGAARD, S. Testing for Sphericity in Pahse I Control Chart Applications. Quality and reliability Engineering International, Vol. 25, p. 839-849, 2009.

WU, Z., JIAO, J., HE, Z. A single control chart for monitoring the frequency and magnitude of an event. International Journal of Production Economics, v. 119, n. 1, p. 24-33, 2009.

ZANTEK, P. F. Design of Cumulative Sum Schemes for Start-Up Processes and Short Runs. Journal of Quality Technology, Vol. 38, No. 4, 2006.

ZHANG, J.; LI, Z.; WANG, Z. Control chart based on likelihood ratio for monitoring linear profiles. Computational Statistics and data Analysis, Vol. 53, p. 1440-1448, 2009.

ZHANG, J.; ZOU, C.; WANG, Z. Control Chart Based on Likelihood Ratio Test for Monitoring Process Mean and variability. Quality and Reliability Engineering International, Vol. 26, p. 63-73, 2010.

ZHOU, Q.; LUO, Y., WANG, Z. A control chart based on likelihood ratio test for detecting patterned mean and variance shifts. Computational and data Analysis, vol. 54, p.1634-1645, 2010.

ZHOU, Z-J.; HU, C-H.; XU, D-L.; CHEN, M-Y.; ZHOU, D-H. A model for real-time failure prognosis based on hidden Markov model and belief rule base. European Journal of Operational Research, Article in Press, 2010.

ZHU, Y. D.; WONG, Y. S.; LEE, K. S. Framework of computer-aided short-run SPC planning system. Int. J. Advanced Manufacturing Technology, Vol. 34, p. 362-377, 2007. 